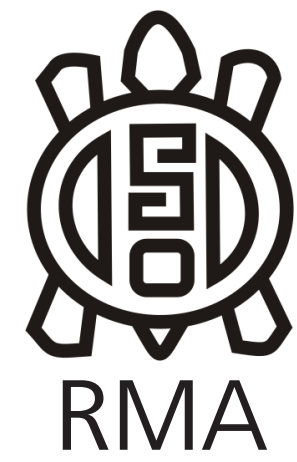

\title{
Perspectivas de análisis integral en el estudio de artefactos líticos
}

\author{
Perspective of integral analysis in the study of lithic artifacts
}

Pilar Babot*, Salomón Hocsman**, Patricia S. Escola†***, María Estela Mansur****

\begin{abstract}
Dossier
Este dossier está dedicado a la memoria de Patricia Escola, querida amiga y colega
\end{abstract}

* Instituto Superior de Estudios Sociales, CONICET / Universidad Nacional de Tucumán

e Instituto de Arqueología y Museo, Facultad de Ciencias Naturales e Instituto Miguel Lillo, UNT. E-mail: pilarbabot@csnat.unt.edu.ar

** Instituto Superior de Estudios Sociales, CONICET / Universidad Nacional de Tucumán e Instituto de Arqueología y Museo, Facultad de Ciencias Naturales e Instituto Miguel Lillo, UNT. E-mail: shocsman@hotmail.com $\dagger^{* * *}$ Centro de Investigaciones y Transferencia de Catamarca, CONICET / Universidad Nacional de Catamarca y Escuela de Arqueología, UNCa. ${ }^{* * * *}$ Centro Austral de Investigaciones Científicas, CONICET y Universidad Nacional de Tierra del Fuego.

E-Mail: estelamansur@gmail.com

y los análisis funcionales de diversa índole, tales como sustancias adheridas, huellas y residuos de uso, a través
Los estudios arqueológicos de artefactos líticos tallados y manufacturados o modificados por picado y abrasión, alcanzaron un grado de desarrollo tal en Argentina que, desde distintas perspectivas, han llevado a un interés creciente por la combinación de distintos tipos de análisis para una mejor comprensión de la gestión de las materias primas y de los instrumentos. De esta forma, está en plena conformación un campo que, como estrategia metodológica, propone el reconocimiento y estudio combinado y complementario de variables morfológicas, dimensionales, tecnológicas, tipológicas, funcionales, cinemáticas y simbólicas que permiten abordar los modos de hacer y de usar desde una perspectiva holística.

Así, los primeros abordajes de esta clase enmarcados en la arqueología contemporánea articularon estudios tecno-tipológicos con el análisis de huellas de uso (Mansur 1983, 1986; Castro 1987-88; entre otros), con el estudio de microfósiles, ácidos grasos o residuos inorgánicos, incorporando la mirada etnoarqueológica y/o experimental (Babot 1999, 2004; Pérez 2004; entre otros), y la caracterización geoquímica de las rocas talladas y sus fuentes (Escola 2000, 2004; entre otros). Los nuevos escenarios se han expandido al incrementar los estudios del desgaste por uso en una diversidad de artefactos líticos tallados y manufacturados o modificados por picado y abrasión (Landini et al. 2000; Leipus 2004, 2016; Álvarez 2007, 2009; Cattáneo y Aguerre 2009; De Angelis et al. 2009; Pal 2009; Massigoge y Pal 2011; Lynch 2013; Mansur et al. 2014; Pérez 2014; Lynch y Miotti 2016; Álvarez Soncini 2018; entre otros) y al promover la articulación de la tecno-tipología de detalle de estudios de microfósiles y físico-químicos diversos que permiten una caracterización elemental o estructural con distinto grado de precisión (Babot et al. 2008; Babot 2010; Babot et al. 2010; Babot et al. 2013; Escola et al. 2013; Lema et al. 2012; Flegenheimer et al. 2014; Mazzia et al. 2016; Cattáneo et al. 2017; entre otros). También se han integrado las aproximaciones actualísticas, como la experimentación (Matarrese 2015; Vecchi 2011; Pérez 2014; entre otros) e interpretaciones basadas en las cosmovisiones nativas (p. ej. Haber y Gastaldi 2006). Sumado a ello, y alineados con una tendencia global en relación a la conservación patrimonial, el desafío está en el diseño de programas analíticos no destructivos o microdestructivos.

En este marco, este dossier reúne una serie de estudios de casos y las aplicaciones o desarrollos metodológicos involucrados en ellos, que exponen y discuten las ventajas de la complementariedad y potenciación de las diferentes líneas de evidencia desde una perspectiva integral de abordaje del instrumental lítico. Se trata de una selección de estudios de las más diversas materialidades y perspectivas teóricas y de un amplio espectro geográficotemporal que abarca desde el extremo meridional chileno del Cono Sur americano hasta los Andes Centrales y alcanza Brasil Central.

Consuelo Huidobro y Mathieu Langlais abordan la cadena operativa de las puntas bifaciales del sitio Offing 2, locus 1, situado en el Estrecho de Magallanes, en la Patagonia Austral de Chile. En su aproximación tecno-funcional aportan información acerca de la funcionalidad de las grandes puntas de proyectil lanceoladas tipo Ponsonby, 
a partir de la caracterización macro, meso y microscópica de huellas de uso. Los autores sugieren una utilización polivalente o flexible, tanto como arma y/o como cuchillo, consistente con datos de la etnografía de estas sociedades canoeras. Se trata de artefactos característicos de las poblaciones marítimas de la región hacia los ca. 4.000 años AP.

Pablo Bianchi y Mariela Careaga, por su parte, proporcionan datos iniciales de un programa mayor que busca establecer el uso de instrumentos líticos tallados procedentes del sitio La Gruta 3 en el Macizo del Deseado, provincia de Santa Cruz, Patagonia argentina, durante el Holoceno Tardío, a partir de la caracterización de residuos lipídicos mediante cromatografía gaseosa acoplada a espectrometría de masas. El estudio se basa en un diseño experimental sobre el procesamiento animal que proporciona un análogo moderno para la interpretación de la información funcional de las piezas arqueológicas. Los autores plantean una agenda futura que sumará información de importancia para las ocupaciones de grupos cazadores-recolectores de la región.

También se refieren a las sociedades cazadoras-recolectoras que ocuparon el ámbito actual de la provincia de Santa Cruz, en su Meseta Central, Manuel Cueto, Andrés Iparraguirre y Rafael Paunero. En su estudio de conjuntos líticos de la Cueva 1 del Cerro Tres Tetas correspondientes a las ocupaciones del Holoceno Medio del área, los autores abordan la variabilidad tecno-morfológica en combinación con el paisaje lítico local para analizar las estrategias de producción seguidas en la fabricación de los instrumentos. Cueto et al. sugieren una consistencia entre el caso de estudio y el conocimiento existente sobre la tecnología lítica regional, expresado, por ejemplo, en el abastecimiento de rocas locales, el uso de obsidianas y el incremento en la tecnología laminar, entre otros.

El estudio integral de los conjuntos líticos manufacturados por picado y abradido y modificados por uso de Norpatagonia es objeto de la contribución de Daniela Saghessi. La autora aborda el instrumental del sitio Loma de Los Muertos emplazado en el Valle del Río Negro, en la provincia homónima, desde perspectivas tecno-morfológicas y morfológico-funcionales. El trabajo contribuye al conocimiento de las materias primas utilizadas a partir de sus atributos macroscópicos y a las características del aprovisionamiento lítico para estos fines. Al considerar atributos morfológicos y de uso macroscópicos y registrados a bajos aumentos, también aporta a la comprensión de los principales procesos de producción y uso de estos conjuntos que proceden de ocupaciones residenciales y de enterratorios del Holoceno Tardío.

Florencia Páez, Mónica Berón, Eliana Lucero y Manuel Carrera Aizpitarte proponen el estudio de una infraestructura para la molienda colectiva emplazada en el Cerro de Los Viejos, en el Departamento Caleu
Caleu, al sur de la provincia de La Pampa, en el área de la Pampa Occidental argentina. Se trata de una manifestación singular en la zona, que es abordada desde el concepto de transgeneracionalidad y apelando a la mirada del paisaje en el que se emplaza. Para ello, se integran variables morfológicas macroscópicas relativas a la manufactura y uso de este ítem, datos del análisis de residuos microfósiles y elementos de la localidad de emplazamiento, datados entre ca. 6000 años y tiempos históricos, que remiten a un lugar de retornos reiterados.

El trabajo de Salomón Hocsman y Pilar Babot propone consideraciones sobre una perspectiva de análisis integral caracterizada por ser situacional, multiproxy, no destructiva y contextualizada. Asimismo, comprende un caso de estudio, el abordaje integral de un conjunto de bifaces del sitio Quebrada Seca 3, situado en Antofagasta de la Sierra, provincia de Catamarca, en la Puna Meridional Argentina. Aspectos de la producción y usos de esta clase artefactual esperados y registrados son escudriñados en una selección de artefactos que corresponden a sucesivas ocupaciones datadas entre ca. 5500 y 4500 años AP. Estos objetos son analizados mediante la combinación de múltiples líneas de evidencia que dialogan entre sí, las cuales comprenden el estudio tecno-tipológico de detalle, análisis múltiple de microfósiles por microscopía de polarización, análisis lipídicos mediante cromatografía gaseosa-espectrometría de masas, de adhesivos empleados en el enmangue a través de espectrometría de infrarrojos por transformada de Fourier y la consideración de evidencias contextuales que nos aproximan a su historia de vida.

Jorge Cabral Ortiz, Cecilia Mercuri, Pilar García de Cecco y Federico Mendoza sugieren una mirada holística de un conjunto inusual de objetos líticos ovoides y esféricos pulidos recuperados en un recinto de piedra del sitio Capilla de La Aguada en Cachi, provincia de Salta, que corresponden a los siglos XV-XVII, entre momentos de conquista inca e hispana. Los autores combinan el abordaje arqueológico con las perspectivas locales, al realizar una caracterización tecno-tipológica de los objetos según variables morfológicas, por un lado, y al aportar a las interpretaciones suministradas por los pobladores locales para dicho conjunto mediante un enfoque etnográfico que sugiere una función simbólica no utilitaria y alternativas de manufactura, por el otro.

Dando paso al área de Brasil Central, un conjunto numeroso de hachas líticas talladas que proceden del sitio Piragiba, en el estado de Bahía, analizado integralmente por Henry Luydy Abraham Fernandes, nos sitúa en contextos de las sociedades ceramistas y horticultoras de ambientes tropicales de los siglos IX a XIV de nuestra era, comprendidas en la Tradición Aratú. Estos instrumentos son estudiados desde el enfoque de la tecnología lítica para establecer las técnicas y métodos de producción, los estados técnicos y las transformaciones en otros 
tipos de instrumentos. También se emplean abordajes macrotraceológicos a ojo desnudo para establecer aspectos del uso que se sustentan en experimentos controlados y se cotejan con información etnográfica. Así, se establecen la recurrencia en el proyecto mental tecno-morfológico de esta clase artefactual y en su historia de vida.

El trabajo de Antonio Pérez Balarezo, Pilar Babot y Lucénida Carrión nos transporta a la Costa Central Peruana, sobre la vertiente occidental de los Andes. Los autores abordan la cuestión de la instrumentalización e instrumentación en el análisis tecno-funcional de conjuntos líticos tardíos, ca. 1.800-400 AP, que proceden de nueve sitios monumentales del Complejo Arqueológico Maranga, en Lima, a fin de integrar las esferas de producción y funcionamiento de los útiles. El diseño metodológico integra diferentes técnicas especializadas que incluyen la petrografía, difracción de rayos $X$, tafonomía, tecnología y análisis de microvestigios vegetales, a fin de identificar los esquemas operatorios de producción y funcionamiento recurrentes en cada conjunto lítico. Los resultados obtenidos se integran e interpelan en un estudio sincrónico y diacrónico de la variabilidad instrumental en el área trabajada.

Para finalizar, es preciso agradecer a los autores que contribuyeron con sus investigaciones a componer un panorama sobre perspectivas integrales en el estudio de artefactos líticos, que ilustra los intereses actuales de este tipo de indagaciones en América del Sur. Un compendio de casos tan diversos no hubiera sido posible sin la mirada crítica y multifacética de los evaluadores quienes, desde diferentes perspectivas y áreas de especialidad, aportaron sus comentarios y observaciones a cada uno de los trabajos que integran este dossier. Un agradecimiento especial a los editores de la Revista del Museo de Antropología de Córdoba por brindar su espacio para difundir los diálogos disciplinares que aquí se incluyen.

\section{Bibliografía}

Álvarez, M.R. 2007. Procesos de producción y uso de instrumentos bifaciales entre los grupo canoeros del Canal Beagle. En: Arqueología de Fuego-Patagonia. Levantando piedras, desenterrando huesos... y develando arcanos, editado por F. Morello, M. Martinic, A. Prieto y G. Bahamonde, pp. 247255. Ediciones CEQUA. Punta Arenas.

Álvarez, M.R. 2009. Diseño y función. Variabilidad instrumental en la costa Norte del Canal Beagle. En: Arqueología Argentina en los Inicios del Nuevo Siglo, editado por C. Gradin y F. Oliva, Tomo II, pp. 293-306. Escuela de Antropología, Facultad de Humanidades y Artes, Universidad Nacional de Rosario. Laborde Editor. Rosario.

Álvarez Soncini, M.C. 2018. Tecnologías de piqueteamiento y pulimentación en la dinámica socio-económica de sociedades cazadoras-recolectoras. Tesis Doctoral, Facultad de Ciencias Naturales y Museo, Universidad Nacional de La Plata. Buenos
Aires, Argentina.

Babot, M.P. 1999. Un estudio de artefactos de molienda. Casos del Formativo. Tesina de grado, Facultad de Ciencias Naturales e Instituto Miguel Lillo, Universidad Nacional de Tucumán. San Miguel de Tucumán, Argentina.

Babot, M.P. 2004. Tecnología y utilización de artefactos de molienda en el Noroeste prehispánico. Tesis de postgrado, Facultad de Ciencias Naturales e Instituto Miguel Lillo, Universidad Nacional de Tucumán. San Miguel de Tucumán, Argentina.

Babot, M.P. 2010. Almidones y fitolitos: desentrañando el papel funcional de los artefactos de molienda arqueológicos. En: Arqueología argentina en los inicios de un nuevo siglo, compilado por F. Oliva, N. de Grandis, y J. Rodríguez, Tomo III, pp. 665-673. Laborde Editor, Rosario.

Babot, M.P., R.G. Cattáneo y S. Hocsman 2010. ¿Puntas de proyectil o cuchillos? Múltiples técnicas analíticas para una caracterización funcional de artefactos arqueológicos. En: La arqueometría en Argentina y Latinoamérica, editado por S. Bertolino, G.R. Cattaneo y A.D. Izeta, pp.127-134. Editorial de la Facultad de Filosofía y Humanidades, Universidad Nacional de Córdoba. Córdoba.

Babot, M.P., P.S. Escola y S. Hocsman 2008. Microfósiles y atributos tecno-tipológicos: Correlacionando raederas de módulo grandísimo con sus desechos de talla de mantenimiento en el Noroeste argentino. En: Matices interdisciplinarios en estudios fitolíticos y de otros microfósiles, editado por M.A. Korstanje y M.P. Babot, pp. 187-200. British Archaeological Reports (BAR) International Series 1870. Hedges. Oxford.

Babot, M.P., S. Hocsman y G.R. Cattáneo 2013. Assessing the life history of projectile points or knives from Middle Holocene of Southern Puna of Argentina. Quaternary International 287: 3-19.

Castro, A. 1987-88. Análisis microscópico de huellas de utilización en artefactos líticos de Fortín Necochea. Paleoetnológicas 4: 65-78.

Cattáneo, G.R. y A.M. Aguerre 2009. Estudios funcionales de artefactos líticos de Cueva de Las Manos, Río Pinturas, Santa Cruz, Argentina. Revista del Museo de Antropología 2: 3-22.

Cattáneo, G.R., M. Martinelli, A. Izeta, J.M. Caminoa y A. Robledo 2017. On wedges and bones: archaeological studies of use-wear and residue analysis from Late Holocene occupations in the Southern Pampean Hills (Alero Deodoro Roca, Córdoba, Argentina). Journal of Archaeological Science Reports 14: 275-288.

De Angelis H., A. Lasa, M.E. Mansur, L. Sosa y G. Valdez 2009. Análisis tecnológico yfuncional de artefactos de vidrio: resultados de un programa experimental. En: Arqueometría Latinoamericana, editado por O. Palacios, C. Vázquez, T. Palacios e E. Cabanillas, pp. 134-141. Comisión 
Nacional de Energía Atómica. Buenos Aires.

Escola, P.S. 2000. Tecnología Lítica y Sociedades Agro-pastoriles Tempranas. Tesis Doctoral, Facultad de Filosofía y Letras, Universidad de Buenos Aires. Buenos Aires.

Escola, P.S. 2004. Variabilidad en la explotación y distribución de obsidianas en la Puna Meridional Argentina. Estudios Atacameños 28: 9-24.

Escola, P.S., S. Hocsman y M.P. Babot 2013. Entre las residencias y los campos de cultivo. Aporte de los cuchillos/raederas de módulo grandísimo a la cuestión del laboreo agrícola en Antofagasta de la Sierra (Puna de Catamarca) durante el primer milenio d.C. Relaciones de la Sociedad Argentina de Antropología XXXVIII(1): 83-110.

Flegenheimer, N., N. Mazzia y M.P. Babot 2013. Estudios de detalle sobre una piedra discoidal pampeana. Intersecciones en Antropología 14: 499-505. I

Haber. A. y M.R. Gastaldi 2006. Vida Con Palas. ANTIPODA, Revista de Antropología y Arqueología 2: 275-302.

Landini, C., M. Bonomo, M. Leipus y G. Martínez, G. 2000. Forma y función de los instrumentos líticos del sitio Paso Otero 3 (pdo. de Necochea, pcia. de Buenos Aires, Argentina): un estudio comparativo. Espacio, Tiempo y Forma, Serie I, Prehistoria y Arqueología 13: 161-187.

Lema, V., C. Della Negra y V. Bernal 2012. Explotación de recursos vegetales silvestres y domesticados en Neuquén: implicancias del hallazgo de restos de maíz y algarrobo en artefactos de molienda del Holoceno tardío. Magallania 40(1): 229-249.

Leipus., M. 2004. Tendencias en el uso de artefactos líticos de la subregión pampa húmeda: relación entre morfología y función a partir del análisis de rastros de utilización. En: La Región pampeana. Su pasado arqueológico, editado por: C. Gradin y F. Oliva, pp. 123-130. Venado Tuerto, Santa Fe.

Leipus, M. 2016. Variabilidad tecnomorfológica y funcional de las raederas en la Región Pampeana (Argentina). Revista de Antropología del Museo de Entre Ríos 2(2): 47-67.

Lynch, V. 2013. Análisis morfológico-funcional de raspadores líticos del sitio cueva Maripe (Santa Cruz, Argentina). En: Tendencias teórico-metodológicas y casos de estudio en la arqueología de la Patagonia, compilado por A.F. Zangrando, R. Barberena, A. Gil, G. Neme, M. Giardina, L. Luna, C. Otaola, S. Paulides, L. Salgán, y A. Tivoli, pp. 417-424. Altuna Impresores, Buenos Aires.

Lynch, V. y L.L. Miotti 2016. Morphological and functional variability on the endscrapers in Cueva Maripe Site (Santa Cruz Province, Argentina). Lithic Technology 41: 60-74.

Mansur, M.E. 1983. Scanning electron microscopy of dry hide working tools: the role of abrasives and humidity in microwear polish formation. Journal of Archaeological Science 10: 223-230.

Mansur, M.E. 1986. Microscopie du matériel lithique préhistorique. Traces d'utilisation, altérations naturelles, accidentelles et technologiques. Traces d'utilisation, altérations naturelles, accidentelles et technologiques. Exemples de Patagonie. Cahiers du Quaternaire $N^{\circ}$ 9, Centre Régional de Publication de Bordeaux, Centre National de la Recherche Scientifique. Éditions du Centre National de la Recherche Scientifique, Paris.

Mansur, M.E., M.A. Lima y Y. Maigrot 2014. Traceology Today: Methodological Issues in the Old World and the Americas. Proceedings of the XVI World Congress (Florianópolis, 4-10 September 2011). Union Internationale des Sciences Préhistoriques et Protohistoriques. BAR International Series 2643. Archaeopress. Oxford.

Massigoge, A. y N. Pal 2011. Producción y uso de artefactos líticos en contextos cazadores-recolectores del Área Interserrana (Argentina): análisis integral de la diversidad tecno-morfológica y funcional. Revista Española de Antropología Americana 41(1): 51-73.

Matarrese, A. 2015. Tecnología lítica entre los cazadoresrecolectores pampeanos: los artefactos formatizados por picado y abrasión y modificados por uso en el área Interserrana Bonaerense. Tesis Doctoral, Facultad de Ciencias Naturales y Museo, Universidad Nacional de La Plata. La PLata, Argentina.

Mazzia, N., C. Weitzel y H. De Angelis 2016. De usos y recursos. Análisis funcional y de sustancias grasas mediante cromatografía gaseosas sobre artefactos líticos tallados. Revista del Museo de Antropología 9(2): 37-50.

Pal, N. 2009. Correlación entre forma-función de los conjuntos líticos recuperados en la cuenca superior del arroyo Tapalqué (Pcia. de Buenos Aires): Su vinculación con otros sitios de la región pampeana. En: Mamül Mapu: pasado y presente. Perspectivas de la arqueología pampeana a comienzos del tercer milenio, editado por M.A. Berón et al., pp. 309-322. Libros del Espinillo, Ayacucho.

Pérez, S. 2004. Experimentación de uso con palas y/o azadas líticas. Intersecciones en Antropología 5: 105-117.

Pérez, S. 2014. La organización de la tecnología lítica en el Noroeste argentino. Aproximación a través de experimentación, análisis tecno-morfológico y de microdesgaste por uso de palas y/o azadas líticas. Tesis doctoral, Facultad de Filosofía y Letras, Universidad Nacional de Buenos Aires, Buenos Aires, Argentina.

Vecchi, R. 2011. Bolas de boleadora en los grupos cazadoresrecolectores de la pampa bonaerense. Tesis Doctoral, Facultad de Filosofía y Letras, Universidad Nacional de Buenos Aires, Buenos Aires, Argentina. 